\title{
House Price Prediction using Machine learning and Image Processing
}

\author{
Jeevan chougale ${ }^{1}$, Abhishek \\ Shinde ${ }^{2}$, Ninad Deshmukh ${ }^{3}$, Dhananjay Sawant ${ }^{4}$, Vaishali Latke ${ }^{5}$ \\ ${ }^{I}$ Student, (Computer Engineering/University of Pune ,Maharashtra INDIA) \\ ${ }^{2}$ Student, (Computer Engineering/University of Pune ,Maharashtra INDIA) \\ ${ }^{3}$ Student, (Computer Engineering/University of Pune ,Maharashtra INDIA) \\ ${ }^{4}$ Student, (Computer Engineering/University of Pune, Maharashtra INDIA) \\ ${ }^{5}$ Asst. Professor (Computer Engineering/University of Pune, Maharashtra INDIA) \\ Emails \\ jeevanchougale4@gmail.com \\ sdabhishek818@gmail.com \\ sawantdhananjay07@gmail.com \\ ninaddeshmukh72@gmail.com \\ vaishali.latke@pccoer.in
}

\begin{abstract}
We demonstrate that these urban features can be recorded by street views and satellite image data and enhance the estimate of house prices. In order to estimate house prices in London, UK, we recommend a pipeline that uses a deep neural network model to automatically extract visual features from images. In calculating the house price model, we use typical housing characteristics, such as age, size and accessibility, as well as visual features from Google Street View images and Bing aerial pictures. We see promising outcomes where learning to describe a neighborhood's urban efficiency facilitates the estimation of house prices, even when generalising to previously unseen London boroughs. We discuss the use of non-linear vs. linear approaches to combine these signals with traditional house pricing models and explain how the interpretability of linear models helps one to specifically derive the visual desirability of neighbourhoods as proxy variables that are both of importance in their own right and can be used as inputs to other econometric methods. This is particularly useful as it can be extended elsewhere after the network has been trained with the training data, enabling us to produce vivid complex maps of the desirability of London streets.
\end{abstract}

Key Word: regression; predictive; artificial; cost-effective; image-processing

\section{Introduction}

It is unsurprising, from an economic standpoint, that citizens are willing to pay for intangible properties. The urban climate directly impacts the societal, economic and health outcomes of individuals. The architecture of a window location will impact the amount of nature seen from inside a home as well as the perceived protection of a street[16]. The amount of greenery will affect all street level contaminants and its scenicity and atmosphere[31]. These variations in the urban landscape are mirrored in the changing rates at which people are willing to pay in the housing market, while other considerations, such as size and access to work, remain constant[6, 18]. Any urban characteristics, such as the operation of a street frontage, the quantity of greenery or the breadth of the pavement, are directly identifiable from images. Others, like the reputation of the neighbourhood or the street's architectural architecture, are less clearly quantifiable. There is a clear lack of analysis, programming resources and data that can be used to uncover these attributes and inform urban planning strategies, considering the close correlation between urban design attributes and economic value. To date, the debate of which features of urban architecture contribute to safer cities or higher prices of property has been largely theoretical, backed quantitatively by just a handful of research. To measure these urban quality metrics requires many street level surveys and structured interviews with professionals. 


\section{Literature Survey}

1. Paper: Housing Price Prediction using Machine Learning Algorithms: The Case of Melbourne City, Australia Author-The DanhPhan Macquarie University Sydney, Australia danh.phanthe@students.mq.edu.au Description: House price forecasting is an important topic of real estate. The literature attempts to derive useful knowledge from historical data of property markets. Machine learning techniques are applied to analyze historical property transactions in Australia to discover useful models for house buyer and sellers..

2. Paper: Predicting the Housing Price Direction using Machine Learning Techniques Author-Debanjan Banerjee Department of Management Information Systems SarvaSiksha Mission Kolkata, India debanjanbanerjee2009@gmail.com Description: Development of civilization is the foundation of increase of demand of houses day by day. Accurate prediction of house prices has been always a fascination for the buyers, sellers and for the bankers also. Many researchers have already worked to unravel the mysteries of the prediction of the house prices. There are many theories that have been given birth as a consequence of the research work contributed by the various researchers all over the world.

3. Paper-Real Estate Value Prediction Using Linear Regression

Author-Nehal N Ghosalkar Department Of Computer Engineering Sardar Patel Institute of Technology Maharashtra, India nehal.ghosalkar@spit.ac.in Description-The study of real estate value is felt critical to help the choices in urban arranging. The land framework is a precarious stochastic process. Financial specialists choices depend on available patterns to procure most extreme returns. Designers are intrigued in knowing the future patterns for their basic leadership. To precisely gauge real estate costs what's more, future patterns, vast measure of information that impacts arrive cost is required for examination, demonstrating and determining.

4. Paper-A Hybrid Regression Technique for House Prices Prediction Author-Sifei Lu, Zengxiang Li, Zheng Qin, Xulei Yang, Rick SiowMongGoh Description-

How to use machine learning algorithms to predict house price? It is a challenge to get as closely as possible result based on the model built. For a specific house price it is determined by location, size, house type, city, country, tax rules, economic cycle, population movement, interest rate, and many other factors which could affect demand and supply. For local house price prediction, there are many useful regression algorithms to use. For example, support vector machines (SVM), Lasso (least absolute shrinkage and selection operator) [2], Gradient boosting [3], Ridge, Random forest. We will investigate and explore them 


\section{Algorithm Explanation}

Convolutional Neural Network is one of the main categories to do image classification and image recognition in neural networks. Scene labeling, objects detections, and face recognition, etc., are some of the areas where convolutional neural networks are widely used.

CNN takes an image as input, which is classified and process under a certain category such as dog, cat, lion, tiger, etc. The computer sees an image as an array of pixels and depends on the resolution of the image. Based on image resolution, it will see as $\mathrm{h} * \mathrm{w} * \mathrm{~d}$, where $\mathrm{h}=$ height $\mathrm{w}=$ width and $\mathrm{d}=$ dimension. For example, An RGB image is 6 * $6 * 3$ array of the matrix, and the grayscale image is $4 * 4 * 1$ array of the matrix.

In CNN, each input image will pass through a sequence of convolution layers along with pooling, fully connected layers, filters (Also known as kernels). After that, we will apply the Soft-max function to classify an object with probabilistic values 0 and 1.

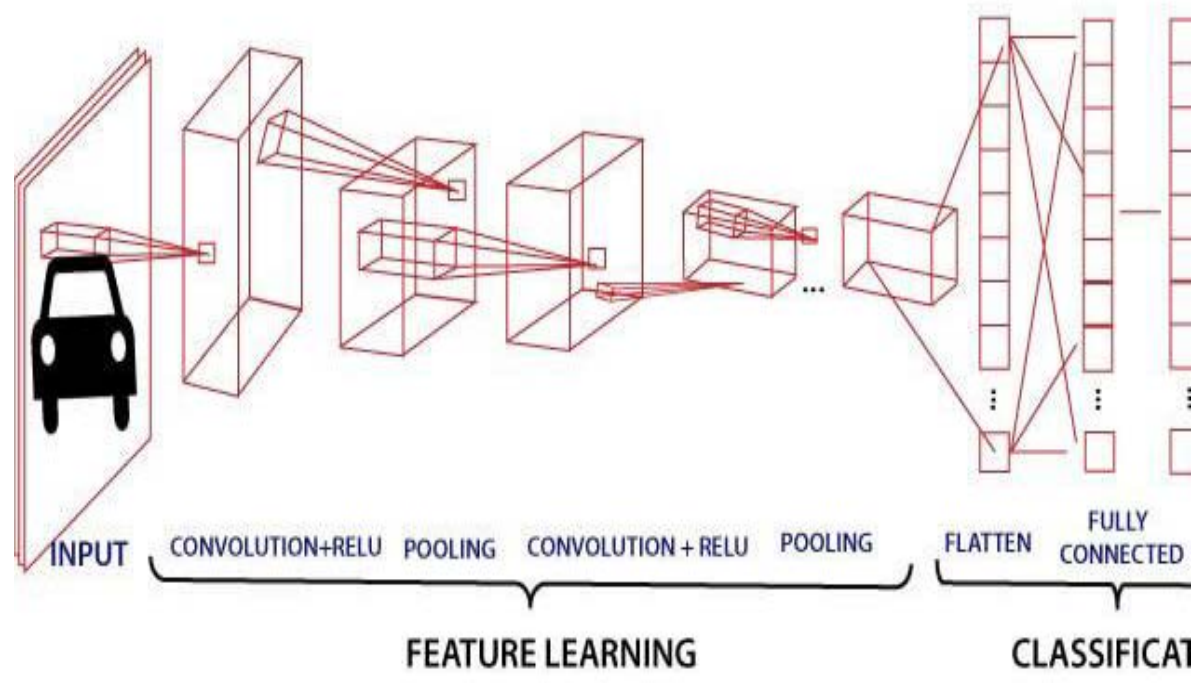

Fig. Algorithm Explanation 


\section{Proposed System}

The following section will outline the current status of machine learning techniques in house price estimation. The first is a study from Peterson and Flanagan [26] that used a multi-layer perceptron model to estimate house price with traditional housing features such as age, size, accessibility and safety. The author compared an artificial neural network hedonic price model with two hidden layers to a standard OLS hedonic price model. The author found significant improvements in the use of an ANN model. The improvements are unsurprising due to the expected nonlinear relations captured by variables in the hidden layers which can not be modeled by a linear OLS model.

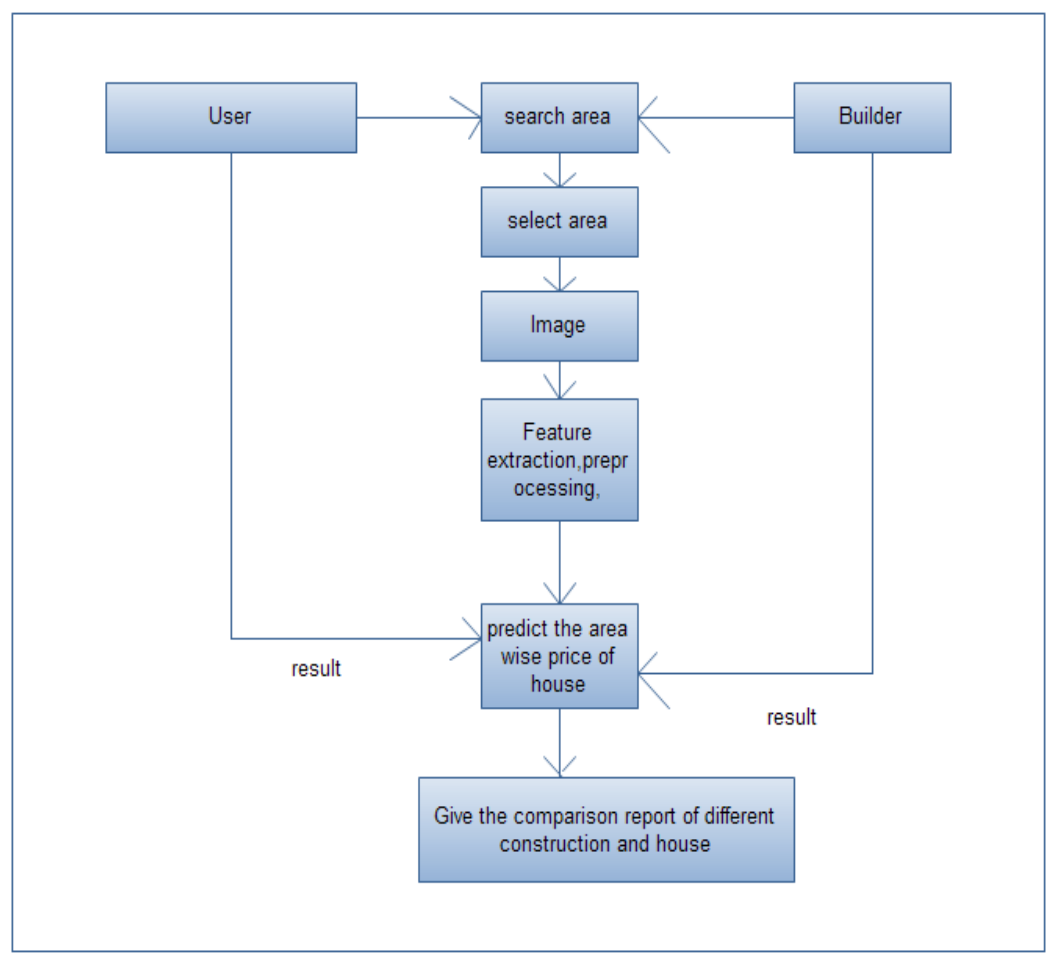

Fig. Proposed System

\section{Conclusion}

visual knowledge of the urban environment to improve predictive power. In contrast to previous work that have made use of images of the interior and exterior of the property for sale, we have focused on characterizing the neighbourhood of the property, and with the property making up only a small proportion of the aerial images; while the Street View images we make use of typically do not contain the property itself. 


\section{References}

[1] Martín Abadi, AshishAgarwal, Paul Barham, Eugene Brevdo, Zhifeng Chen, Craig Citro, Greg S. Corrado, Andy Davis, Jeffrey Dean, Matthieu Devin, Sanjay Ghemawat, Ian

(2015). https://www.tensorflow.org/ Software available from tensorflow.org.

[2] E Ahmed and M Moustafa. 2016. House price estimation from visual and textual features. arXiv:1609.08399[cs.CV] (2016).

[3] S Arietta, Afros, R Ramamooorthi, and M Agrawala. 2014. City Forensics: Using Visual Elements to Predict Non-Visual City Attributes. IEEE Transactions on Visualization and Computer Graphics (2014).

[4] Herbert Bay, TinneTuytelaars, and Luc Van Gool. 2006. Surf: Speeded up robust features. In ECCV.404-417.

[5] L Chen, G Papandreou, I Kokkinos, K Murphy, and A.L. Yuille. 2014. Semantic image segmentation with deep convolutional nets and fully connected crfs. arXiv preprint arXiv:1412.7062 (2014).

[6] J Cheshire and S Sheppard. 1995. On the Price of Land and the Value of Amenities. Economica (1995).

[7] François Chollet. 2015. keras. https://github.com/fchollet/keras. (2015).

[8] C Doersch, S Singh, C Wu, and W Hui. 2012. ACM Transactions on Graphics. What makes Paris look like Paris (2012).

[9] A Dubey, N Naik, D Parikh, R Raskar, and C Hidalgo. 2016. Deep Learning the City : Quantifying Urban Perception At A Global Scale. European Conference on Computer Vision (ECCV) (2016).

[10] Historic England. 2017. https://historicengland.org.uk/listing/the-list/ data-downloads/. (2017). 